\title{
NUMERICAL MODELING OF HEAT STORAGE IN
} SOILS

\author{
Olaf KOLDITZ $^{1,2}$, Christopher McDERMOTT ${ }^{3}$, \\ Rüdiger WORSCH${ }^{4}$, Philipp BLUM ${ }^{4}$ and Peter GRATHWOHL ${ }^{4}$ \\ ${ }^{1}$ Department of Environmental Informatics, Helmholtz Centre for Environmental Research \\ (Permoser Str. 15, D-04318, Leipzig, Germany) \\ E-mail: olaf.kolditz@ufz.de \\ 2 Applied Environmental System Analysis, Technical University of Dresden \\ ${ }^{3}$ Edinburgh Collaborative of Subsurface Science and Engineering (ECOSSE), School of Geoscience, \\ University of Edinburgh \\ (West Mains Road, Edinburgh, EH9 3JW, Scotland) \\ ${ }^{4}$ Center for Applied Geosciences, University of Tübingen \\ (Sigwartstr. 10, D-72076 Tübingen, Germany)
}

\begin{abstract}
In this paper we present a numerical model for the simulation of non-isothermal air flow and corresponding heat transport in the unsaturated soil zone. The governing equations for non-isothermal gas flow and related heat transport are derived. The thermo-hydraulic $(\mathrm{TH})$ coupling is additionally due to material properties, such as density, viscosity, heat diffusivity, which depends on the primary variables air pressure and temperature. A finite element model is developed for the solution of the resulting initial-boundary-value-problem. The numerical model is verified against analytical solutions for simplified problems. Finally, a case study for heat storage in soils is conducted.
\end{abstract}

Key Words : Geothermal energy, non-isothermal fluid flow, porous media, soils, numerical modeling

\section{INTRODUCTION}

Continuously rising oil and gas prizes and the increase of the greenhouse gases call more and more societal attention to the need for more sustainable energy resources. The growing consumption of fossil fuels put increasingly pressure on the current natural resources and environment (e.g. 1)). Today China and the United States of America consume around 40 percent of today's primary energy. China's oil consumption, for example, soared by 11 percent in 2004 , cementing its position as the world's number two user after the USA with 6.6 million barrels per day $^{2}$. Hence, a sustainable global energy supply and a more efficient use of energy are essential for our future climate and generations.

One of the sustainable energy resources is the heat that is stored in the earth, which is an inexhaustible source of energy and could be used almost anywhere in the world. However, currently solar and geothermal energy technologies contribute only around 0.4 percent to the global primary energy supply ${ }^{3}$. Both technologies and the combination of both however would have the potential to decrease the consumption of fossil fuels for electricity and heating. Hence, in the current study a combined application of both techniques, the storage of heat in unsaturated soils and hard rocks by pumping solar-heated air into soils, is considered. Only little research has been performed on the heat storage in the unsaturated zone. For example, 4) described two model experiments to evaluate heat storage in the unsaturated zone. Hot water was supplied to an insulated steel tank, which was filled at the top with soil underlain by weathered chalk. Temperature distribution in the tank was measured by thermo-couples to get information on the chalks ability to exchange heat from flowing water and its capacity to store heat. In the first experiment after 100 hours of injecting water 51 percent of the stored heat in water was actually stored in the tank. In the second experiment a glasshouse was placed on a 
tank, air and soil temperatures were measured. A total of 38 percent of the supplied heat were stored in the chalk after the injection period. Air temperature of the glasshouse was also significantly affected. 5) studied the heat storage in unsaturated soils in warm and semi-arid climatic zones. A field experiment, where a helical heat exchanger was inserted into the soil, was conducted in Israel. Warm water $\left(65-70{ }^{\circ} \mathrm{C}\right)$ was pumped into the heat exchanger for 9 months, depositing heat in the surrounding soil. For heat extraction from the soil, water with $20 \mathrm{C}$ was pumped through the heat exchanger for a period of one month. Modeling of the experiment was done with the computer code PT considering fully coupled heat and liquid flow ${ }^{6}$. A third study was performed by 7), who carried out a field experiment in Finland in 1990 to 1992 . Several wells were drilled and ambient air was pumped into the soil in summer time and extracted in winter time. Evolution of pressure and temperature was measured in observation wells. Evaluation of these field experiments showed that thermal energy storage in cold climatic zones is technically feasible, however due to low temperature of the air pumped into the soil economical not profitable. 8) simulated the field experiment using the multi-phase multi-component model TOUGH ${ }^{9}$ and the single-phase model THETA ${ }^{10)}$. A good agreement with the temperature and pressure data from the field was found after the calibration of the multi-phase model. The results from the single-phase model showed a deviation from only $1-2{ }^{0} \mathrm{C}$ compared to the multi-phase model.

Gas flow models are applied in the field of reservoir management of natural gas reservoirs, well testing, vadose zone hydrology, and soil physics, e.g. for exploration of gas containing formations (characterization of the gas reservoirs permeability, evaluation of the resources), testing of the hydraulic characteristics of low-permeable rock, evaluation of the air-permeability in the unsaturated soil zone, and contaminant transport as well as aquifer remediation by pneumatic techniques. Frequently, air injection and extraction techniques are used to determine the in-situ hydraulic characteristics of the subsurface. By this way, the observed pressure build-up or drawdown during the pneumatic well testing is used to evaluate the air- permeability of soil or rock. Different types of experiments exist such as pulse or interference tests. 11) discussed the special usage of different interpretation methods such as Muskat's approach, and the Theis-Horner method.

In contrast to groundwater hydraulics, air flow is more complicated because of its compressibility. Significant variations in air density and viscosity can result also from temperature fluctuations (so-called Klinkenberg effect). According to the kinetic theory of gases, its viscosity should not depend on the pressure. This is not necessarily the case for conditions typically existing in natural gas reservoirs. At a fixed temperature, the viscosity of gas can vary by tens of percents as the formation pressure changes by a few Mega Pascals. Another problem concerns the evidence of turbulent flow which results in additional friction effects. Turbulent terms are present in the equation of momentum conservation (Darcy's law).

The theory of gas seepage was developed first by 12) 13) 14), who worked out a number of analytical approximations to solve the nonlinear problem. Other analytical solutions for analyzing gas flow in the unsaturated zone were developed in the last decades by 15) 16) 17) 18) 19) 20) 21). Numerical gas flow models were developed by 22) 23) 24) 25) 26) 27) 28) 29). To this end, a number of general assumptions must be introduced, e.g. gravitational forces are neglected, no phreatic surfaces are formed, and idealized material properties must be assumed. The state of the compressible fluid within a considered closed system may be isothermal (const. temperature), adiabatic (const. heat content), or polytropic (const. change of heat content).

\section{GOVERNING EQUATIONS}

In this section we, first, develop the governing equations for air flow and related heat transport in porous media (section 2.(1)). Second, hydromechanical and thermodynamical properties of air depending on gas pressure and temperature are given (section 2.(3)).

\section{(1) Air flow}

The equation of air flow in a porous medium can be derived from the mass balance of fluid (gas) mass

$$
\frac{\partial \rho^{a}}{\partial t}+\nabla \cdot\left(\rho^{a} \mathbf{v}\right)=Q_{\rho}
$$

where $\rho^{\alpha}$ is air density, $\mathbf{v}$ is velocity vector, $Q_{\rho}$ is a fluid mass density source/sink term. The equation of state for an ideal gas (2) represents its compressibility and expansivity due to pressure and temperature changes, respectively.

$$
\rho^{g}=\frac{p}{R T}
$$

where $\rho^{g}$ is gas denstity, $p$ is gas pressure, $R$ is the gas constant which is for air equal to $R_{\text {air }}=287 \mathrm{~J} /(\mathrm{kgK})$ and $T$ is temperature in Kelvin. Therefore, the air density 
at atmospheric pressure and $T=293 K$ is

$$
\rho^{a}=\frac{101325 P a}{287 J /(k g K) 293 K}=1.20433 \frac{\mathrm{kg}}{\mathrm{m}^{3}}
$$

For isothermal flow, i.e. $T=T_{0}$ we have

$$
\frac{\partial p}{\partial t}+\nabla \cdot(p \mathbf{v})=R T_{0} Q_{\rho}
$$

In addition with the momentum balance equation, which can be expressed in form of an extended Darcy's law for non-linear flow.

$$
n \mathbf{v}=-\frac{\mathbf{k}}{\mu} \nabla p
$$

where $n$ is porosity, $\mathbf{k}$ is permeability tensor, $\mu$ is fluid viscosity, the air mass balance equation reads as

$$
n \frac{\partial p}{\partial t}-\nabla \cdot\left(p \frac{\mathbf{k}}{\mu} \nabla p\right)=n R T_{0} Q_{\rho}
$$

which is a non-linear equation with respect to air pressure $p$.

For non-isothermal flow we have to account for temperature changes in the ideal gas law (2) as well. The fluid mass balance equation is then given by

$$
\begin{array}{r}
n \frac{1}{T} \frac{\partial p}{\partial t}-n \frac{p}{T^{2}} \frac{\partial T}{\partial t}+\frac{p}{T^{2}} \nabla T \cdot \frac{\mathbf{k}}{\mu} \nabla p \\
-\frac{1}{T} \nabla \cdot\left(p \frac{\mathbf{k}}{\mu} \nabla p\right)=n R Q_{\rho}
\end{array}
$$

\section{(2) Heat transport}

For heat transport it is assumed, that the liquid phase is stagnant, i.e. $\mathbf{v}^{l}=0$. Therefore, the advective heat transport is only due to air flow. Thermal effects of the liquid phase are taken into account in heat storage and heat conductivity processes. With this simplification, heat transport in soil can be described by the following equation.

$$
c \rho \frac{\partial T}{\partial t}+c^{g} \rho^{g} n \mathbf{v}^{g} \cdot \nabla T-\lambda \Delta T=Q_{T}
$$

with $c \rho=n\left(S^{g} c^{g} \rho^{g}+S^{l} c^{l} \rho^{b}\right)+(1-n) c^{s} \rho^{s}$, heat capacity of porous medium and $\lambda=n\left(S^{g} \lambda^{g}+S^{l} \lambda^{l}\right)+(1-n) \lambda^{s}$, heat conductivity of porous medium. $S^{l}=1-S^{g}$ is fluid phase saturation. Superscripts $g, l, s$ denote gas, liquid and solid phases of the porous medium, respectively. Using the ideal gas law (2), density of the porous medium is given by

$$
\rho=n S^{g} \frac{p}{R T}+n S^{l} \rho^{l}+(1-n) \rho^{s}
$$

\section{(3) Material functions}

For non-isothermal air flow and heat transport we have to consider in addition to the ideal gas law (2) the pressure and temperature dependencies of air viscosity $\mu^{g}(p, T)$ (section 2.(3)a)), specific heat capacities $c(p, T)$ and heat conductivities $\lambda(p, T)$ (section 2.(3)b)) as well 30).

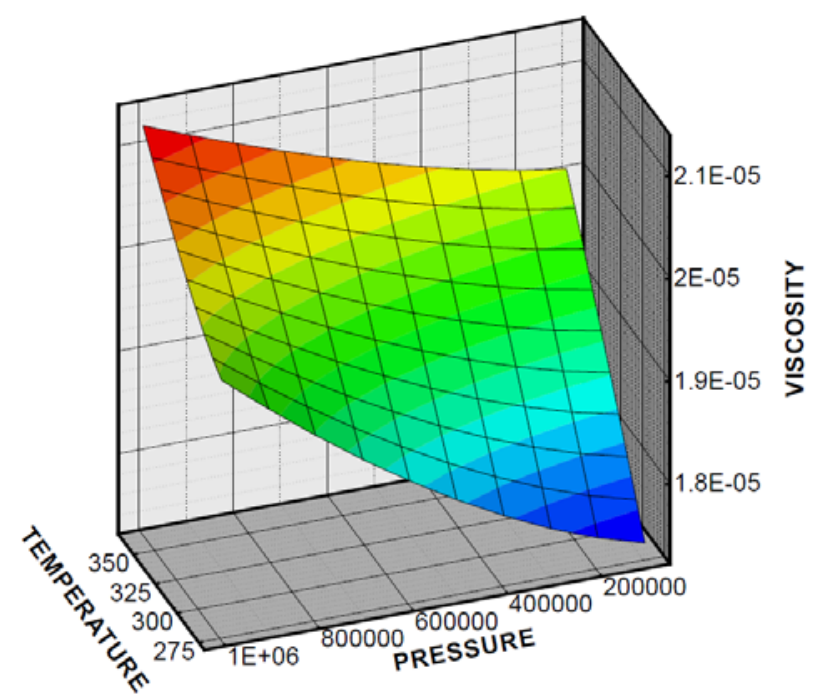

Fig.1 Air viscosity as a function of temperature (in Kelvin) and pressure (in $\mathrm{Pa}$ )

\section{a) Air viscosity}

The Reichenberg viscosity model 31) is used for the non-isothermal flow of air. The pressure and temperature dependencies of air viscosity are shown in Fig. 1.

$$
\mu^{g}(p, T)=\mu_{0}(T)\left(1+\frac{A p_{r}^{3 / 2}}{B p_{r}+\left(1+C p_{r}^{D}\right)^{-1}}\right)
$$

with the following parameters:

$$
\begin{array}{cc}
p_{r}=p / p_{\text {crit }} & T_{r}=T / T_{\text {crit }} \\
A=\frac{\alpha_{1}}{T_{r}} \exp \left(\alpha_{2} T_{r}^{a}\right) & B=A\left(\beta_{1} T_{r}-\beta_{2}\right) \\
C=\frac{\gamma_{1}}{T_{r}} \exp \left(\gamma_{2} T_{r}^{c}\right) & D=\frac{\delta_{1}}{T_{r}} \exp \left(\delta_{2} T_{r}^{d}\right)
\end{array}
$$




$\begin{array}{lll}p_{\text {crit }}=33.9 \times 10^{4} \mathrm{~Pa} & T_{\text {crit }}=126.2 \mathrm{~K} & \\ \alpha_{I}=1.9824 \times 10^{-3} & \alpha_{2}=5.2683 & a=-0.5767 \\ \beta_{I}=1.6552 & \beta_{2}=1.2760 & \\ \gamma_{I}=0.1319 & \gamma_{2}=3.7035 & c=-79.8678 \\ \delta_{I}=2.9496 & \delta_{2}=2.9190 & d=-16.6169\end{array}$

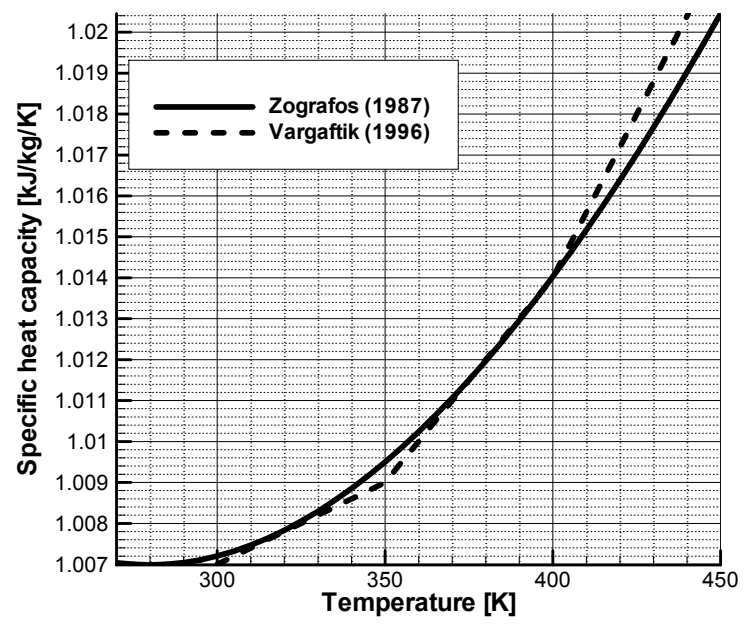

Heat capacity

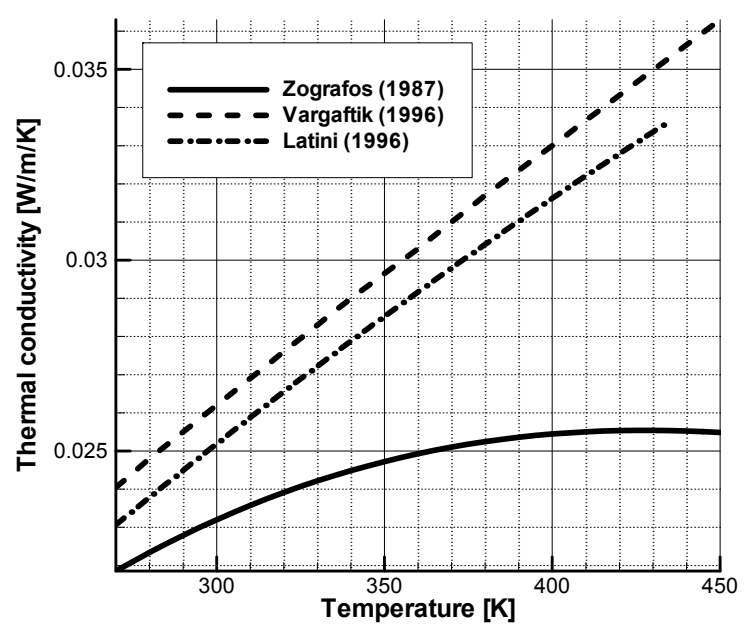

Thermal conductivity

Fig.2 Thermal properties of air, compiled in 36)

\section{b) Thermal properties}

Beside the hydraulic characteristics such as air viscosity, the thermal gas and solid properties such as heat capacity and thermal conductivity are important for heat transport. As an example, Fig. 2 depicts the thermal properties of the gaseous phase. Fig. 2 (left) shows the temperature dependence of specific heat capacity of air at atmospheric pressure corresponding to equation (12) from 32) compared with experimental data by 33 ). Fig. 2 (right) illustrates the temperature dependence of thermal conductivity of air at atmospheric pressure corresponding to equation (13) from 32) compared with experimental data by 33). The pressure dependency of thermal properties can be neglected in the present pressure regimes.

$$
\begin{aligned}
c^{g}=1.0613 & -4.3282 \times 10^{-4} T \\
+ & 1.0234 \times 10^{-6} T^{2} \\
& -6.4747 \times 10^{-10} T^{3} \\
& +1.3864 \times 10^{-13} T^{4} \\
\lambda^{g}=7.488 \times 10^{-3} & -1.7082 \times 10^{-4} T \\
& +2.3758 \times 10^{-7} T^{2} \\
& -2.2012 \times 10^{-10} T^{3} \\
& +9.46 \times 10^{-14} T^{4} \\
& -1.579 \times 10^{-17} T^{5}
\end{aligned}
$$

\section{NUMERICS}

The method of weighted residuals is applied to derive the weak formulations of the governing balance equations given in section 2 . The finite element method (FEM) is used in order to obtain the numerical model for non-isothermal gas flow and related heat transport.

\section{(1) Air flow equation}

Assume $v^{n} \subset H_{\Gamma}^{1}(\Omega)^{n}$ is the test function space, where $H_{\Gamma}^{1}$ is Hilbert space, $\Omega$ is space domain bounded by $\Gamma, n$ is the order of test functions. The mathematical concept of a Hilbert space generalizes the notion of Euclidean space in a way that extends methods of vector algebra from three-dimensional space to infinite-dimensional spaces. For all linear test functions $w \in v^{1}$, we have the weak form of the mass balance equation (1) as

$$
\int_{\Omega}\left(\frac{\partial \rho}{\partial t}+\nabla \cdot(\rho \mathbf{v})-Q_{\rho}\right) w \mathrm{~d} \Omega=0
$$

Applying integration by parts, equation (14) can be rewritten as

$$
\begin{array}{r}
\int_{\Omega} \frac{\partial \rho}{\partial t} w \mathrm{~d} \Omega-\int_{\Omega}(\rho \mathbf{v}) \cdot \nabla w \mathrm{~d} \Omega \\
+\int_{\Gamma}(\rho \mathbf{v}) \cdot \mathbf{n} w \mathrm{~d} \Omega-\int_{\Omega} Q_{\rho} w \mathrm{~d} \Omega=0
\end{array}
$$

Introducing now the ideal gas law according to equation (2) we obtain the following weak form of the fluid mass balance equation. 


$$
\begin{array}{r}
\int_{\Omega} n\left(\frac{1}{T} \frac{\partial p}{\partial t}-\frac{p}{T^{2}} \frac{\partial T}{\partial t}\right) w \mathrm{~d} \Omega \\
-\int_{\Omega}\left(\frac{p}{T} \frac{\mathbf{k}}{\mu} \nabla p\right) \cdot \nabla w \mathrm{~d} \Omega \\
=R \int_{\Omega} Q_{\rho} w d \Omega-\int_{\Gamma}\left(\frac{p}{T} \mathbf{v}\right) \cdot \mathbf{n} w \mathrm{~d} \Omega
\end{array}
$$

In the framework of FEM, unknown function air pressure $p$ is approximated by following trial solution based on nodal values $p_{i}$ and interpolations functions $\phi=\left\{\phi_{1}, \ldots, \phi_{i}, \ldots, \phi_{n}\right\}$

$$
p \approx \hat{p}=\sum_{i}^{n} \phi_{i}(x) p_{i}(t)
$$

where $i$ is node number and $n$ is number of grid nodes. Inserting the function approximations (17) into the weak form of mass balance (16), finally we obtain the following system of algebraic equations.

$$
\mathbf{M}_{p} \frac{d \mathbf{p}}{d t}+\mathbf{M}_{p T} \frac{d \mathbf{T}}{d t}+\mathbf{K}_{p} \mathbf{p}=\mathbf{r}_{p 1}+\mathbf{r}_{p 2}
$$

with nodal vector for pressure

$\mathbf{p}=\left\{p_{1}, \ldots, p_{i}, \ldots, p_{n}\right\}$.The finite element (FE) matrices of equation system (18) are

$$
\mathbf{M}_{p}(T)=\int_{\Omega} \phi \frac{n}{T} w \mathrm{~d} \Omega
$$

Fluid mass matrix

$$
\mathbf{M}_{p T}(p, T)=\int_{\Omega} \phi\left(\frac{p}{T^{2}}\right) w \mathrm{~d} \Omega
$$

Thermal expansion coupling matrix

$$
\mathbf{K}_{T}(p, T)=\int_{\Omega} \nabla \phi\left(\frac{p}{T} \frac{\mathbf{k}}{\mu}\right) \nabla w \mathrm{~d} \Omega
$$

Fluid conductivity matrix

\section{(2) Heat transport equation}

Conducting the same procedure as described above for the fluid mass balance equation (section 3.(1)) we derive the following weak formulation of the heat transport equation (8).

$$
\int_{\Omega}\left(c \rho \frac{\partial T}{\partial t}+c^{g} \rho^{g} n \mathbf{v}^{g} \cdot \nabla T-\lambda \Delta T-Q_{T}\right) w \mathrm{~d} \Omega
$$

Applying integration by parts, equation (20) can be rewritten as

$$
\begin{array}{r}
\int_{\Omega} c \rho \frac{\partial T}{\partial t} w d \Omega+\int_{\Omega} c^{g} \rho^{g} n \mathbf{v}^{g} \cdot \nabla T w \mathrm{~d} \Omega \\
+\int_{\Omega} \lambda \nabla T \cdot \nabla w \mathrm{~d} \Omega \\
-\int_{\Gamma} \lambda \nabla T \cdot \mathbf{n} w \mathrm{~d} \Omega-\int_{\Omega} Q_{T} w \mathrm{~d} \Omega=0
\end{array}
$$

In the framework of FEM, unknown function temperature $T$ is approximated by following trial solution based on nodal values and interpolations functions 34).

$$
T \approx \hat{T}=\sum_{i}^{n} \phi_{i}(x) T_{i}(t)
$$

Inserting the approximations (22) into the weak form of heat transport equation (21), finally we obtain the following system of algebraic equations.

$$
\mathbf{M}_{T} \frac{d \mathbf{T}}{d t}+\mathbf{A}_{T} \mathbf{T}+\mathbf{K}_{T} \mathbf{T}=\mathbf{r}_{T 1}+\mathbf{r}_{T 2}
$$

with nodal vectors for temperature $\mathbf{T}=\left\{T_{1}, \ldots, T_{i}, \ldots, T_{n}\right\}$. The FE matrices of equation system (23) are

$$
\mathbf{M}_{T}(p, T)=\int_{\Omega} \phi(c \rho) w \mathrm{~d} \Omega
$$

Heat mass matrix

$$
\begin{aligned}
& \mathbf{A}_{T}(p, T)=\int_{\Omega} \nabla \phi\left(c \frac{p}{T}\right) w \mathrm{~d} \Omega \\
& \mathbf{K}_{T}(T)=\int_{\Omega} \nabla \phi(\lambda) \nabla w \mathrm{~d} \Omega
\end{aligned}
$$

Heat diffusion matrix

\section{(3) Solution scheme}

We use an partitioned algorithm for the solution of the coupled problem and a fixpoint iteration scheme for the solution of the non-linear flow problem. Fig. 3 illustrates the emplacement of the non-linear and coupling iterations, respectively. 


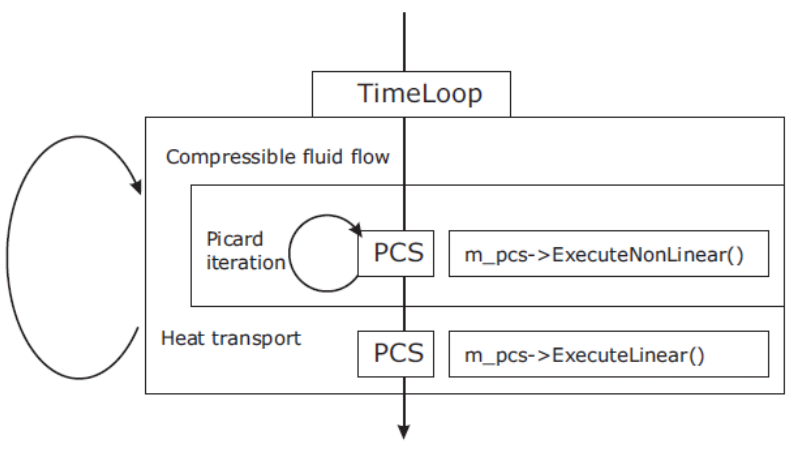

Fig.3 Iteration scheme of the partitioned coupling algorithm

Table 1 Model parameters, 37)

\begin{tabular}{llll}
\hline \hline Property & Symbol & Value & Unit \\
\hline Model length & $L$ & 100 & $\mathrm{~m}$ \\
Cross section area & $A$ & 1 & $\mathrm{~m}^{2}$ \\
Porosity & $n$ & 0.35 & - \\
Densities & $\rho^{g}, \rho^{l}, \rho^{s}$ & $1,1000,2650$ & $\mathrm{~kg} / \mathrm{m}^{3}$ \\
\hline Permeability & $k$ & $2.7 \times 10^{-11}$ & $\mathrm{~m}^{2}$ \\
Dynamic gas viscosity & $\mu$ & $1.76 \times 10^{-5}$ & $\mathrm{~Pa} \mathrm{~s}$ \\
Initial condition & $p_{I}$ & 101325 & $\mathrm{~Pa}$ \\
Boundary condition & $p_{1}$ & 101325 & $\mathrm{~Pa}$ \\
Injection rates & $Q^{p}$ & $1-10$ & $\mathrm{~kg} / \mathrm{s}$ \\
\hline Heat dispersion length & $\alpha_{L}, \alpha_{T}$ & $1,0.1$ & $\mathrm{M}$ \\
Heat conductivities & $\lambda^{g}, \lambda^{l}, \lambda^{s}$ & $0.024,0.6,8.8$ & $\mathrm{~W} / \mathrm{m} / \mathrm{K}$ \\
Heat capacities & $c^{g}, c^{l}, c^{s}$ & $1000,4185,800$ & $\mathrm{~J} / \mathrm{kg} / \mathrm{K}$ \\
\hline Time step & $\Delta t$ & $10^{4}$ & $\mathrm{~s}$ \\
Space step & $\Delta x$ & 1 & $\mathrm{~m}$ \\
\hline \hline
\end{tabular}

First the non-linear air flow equation (7) is solved. The non-linearity arises from the conductivity term $\left(p / T^{2} \nabla T \cdot \mathbf{k} / \mu \nabla p\right)$. Then the heat transport equation (8) is solved, which needs gas flow velocity $\mathbf{v}^{\mathrm{g}}$ as coupling term. As in non-isothermal flows the fluid properties depends on temperature, at every time level an additional coupling iteration loop between both balance equations is necessary.

\section{MODEL VERIFICATION}

Two test examples are presented. The first test case is dealing with flow in porous media of an compressible fluid including temperature dependent fluid properties (sec. 4.(1)). The second examples shows the advantages of object-orientation in finite element implementation (sec. 4.(2)).

\section{(1) Compressible flow test}

We consider a simple test 1-D test example. A one hundred meter long soil column is heated by air injection. One to ten kilogram air per second are injected into the soil. The material parameters are summarized in Table 1.

For isothermal flow with Dirichlet boundary

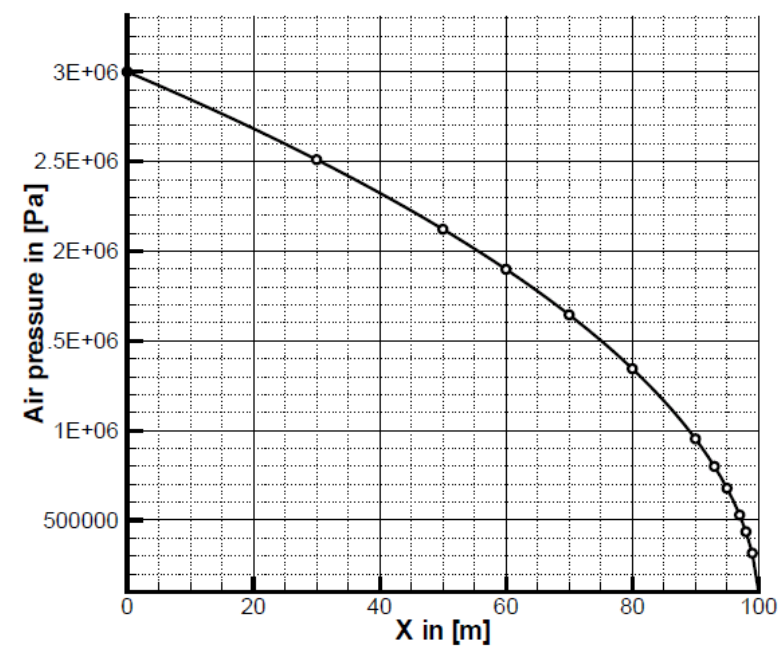

Fig.4 Comparision of analytical (circles) and numerical solution

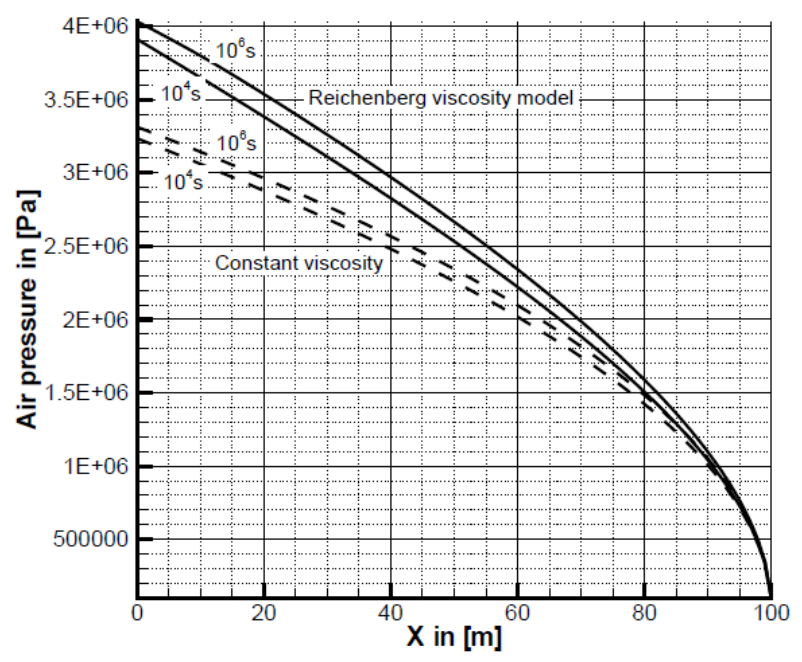

Air pressure profile evolution

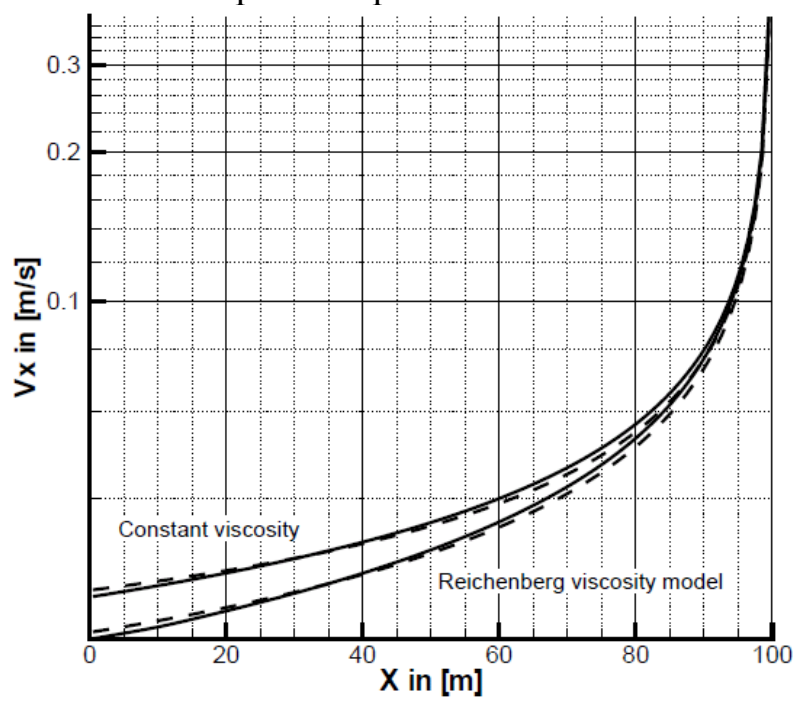

Air velocity profile evolution

Fig.5 Hydraulic profiles evolution

conditions, i.e. $p(0, t)=p_{0}$ and $p(100, t)=p_{1}$, there exists an analytical solution, 


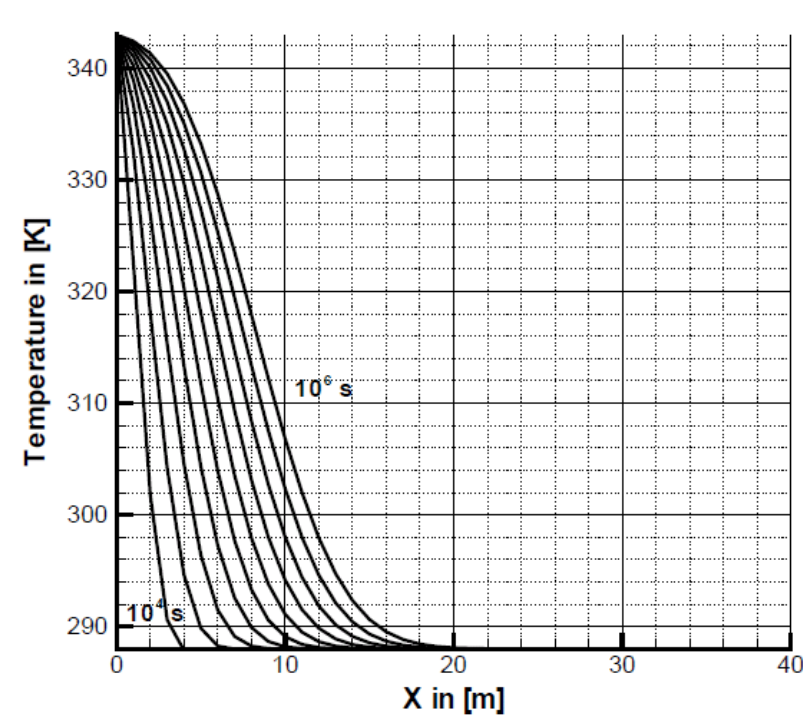

$1 \mathrm{~kg} / \mathrm{s}$ air injection rate

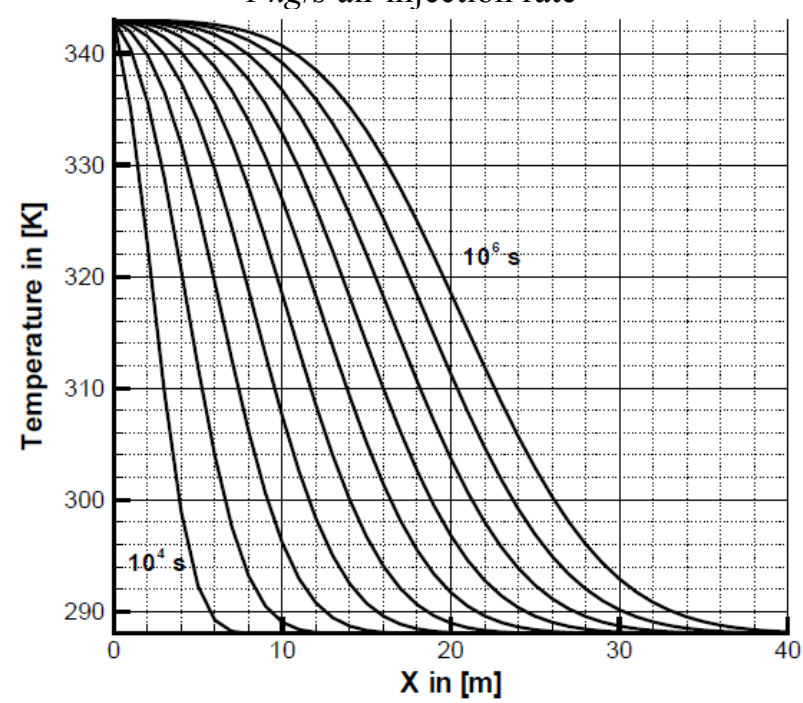

$10 \mathrm{~kg} / \mathrm{s}$ air injection rate

Fig.6 Air temperature profiles evolution

$$
p(x)=\sqrt{\left(p_{0}^{2}-p_{1}^{2}\right) \frac{x}{x_{2}-x_{1}}+p_{1}^{2}}
$$

which is used for verification of the numerical solution. The boundary condition $p_{0}$ is related to the corresponding injection rate $Q^{p}$.

Fig. 4 shows the comparison of analytical and numerical solutions, which compare very well.

Fig. 5 show the air pressure (left) and velocity distributions (right) along the soil profile. Simulations were run with viscosities constant and those corresponding to the Reichenberg model (section 2.(3)a)) which takes pressure and temperature changes into account.

The corresponding temperature profiles for different air injection rates are depicted in Fig. 6. The different shapes of the thermal profile curves indicate
Table 2 Material parameters

\begin{tabular}{lll}
\hline \hline Parameter & Unit & Value \\
\hline \hline Density & $\mathrm{kg} / \mathrm{m}^{3}$ & 1.225 \\
\hline Viscosity & $\mathrm{Pa} \mathrm{s}$ & $1.78 \times 10^{-5}$ \\
\hline Porosity & - & 0.005 \\
\hline Permeability & $\mathrm{m}^{2}$ & $2.77 \times 10^{-19}$ \\
\hline \hline
\end{tabular}

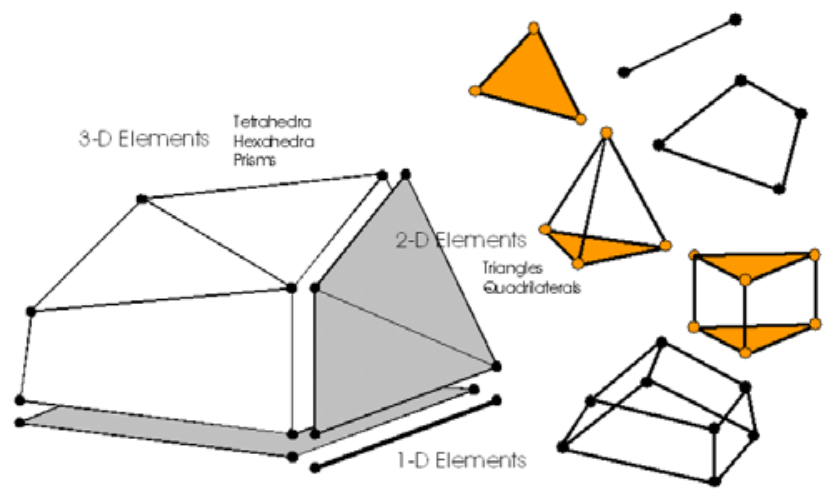

Fig.7 Different element types

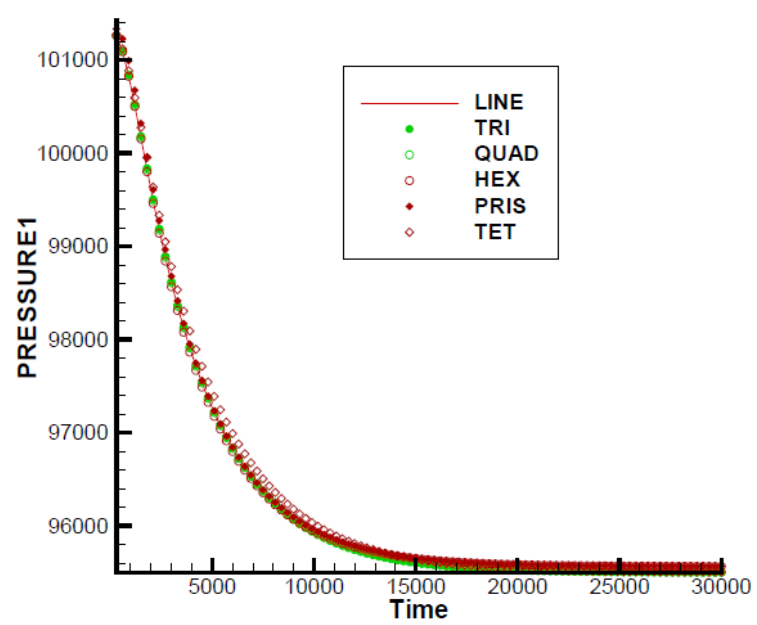

Fig.8 Evolution of gas pressure at observation point

the transition between diffusion (left) and advection dominated regimes (right).

\section{(2) Element test}

This example is presented first of all for code verification of all element types implemented, i.e. lines, triangles, quads, tetrahedra, triangle prisms and hexahedra 35). We consider a non-linear problem, flow of a compressible fluid through a porous medium. In this case the hydraulic conductivity is dependent on fluid pressure.

The discretizations with different element types is shown in Fig. 7. The initial gas pressure distribution is equal to $101325 \mathrm{~Pa}$ everywhere in the model domain. There are Dirichlet boundary condition set at left $p^{g}(x=0 m)=95500 \quad \mathrm{~Pa}$ and right hand side $p^{g}(x=100 m)=101325 \mathrm{~Pa}$, respectively, in order to extract gas from the domain. The material parameters of the fluid and the porous medium are given in 


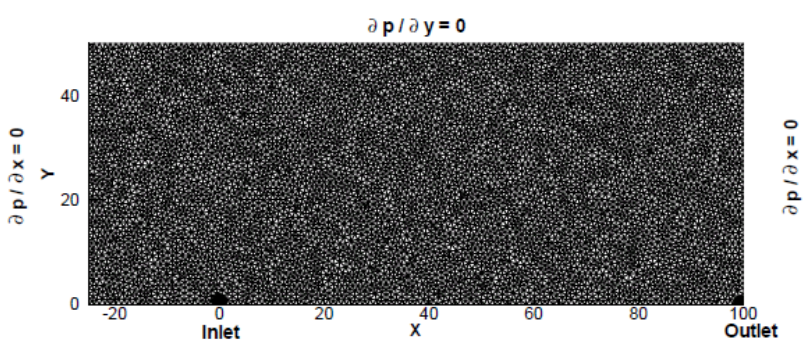

Fig.9 2D problem
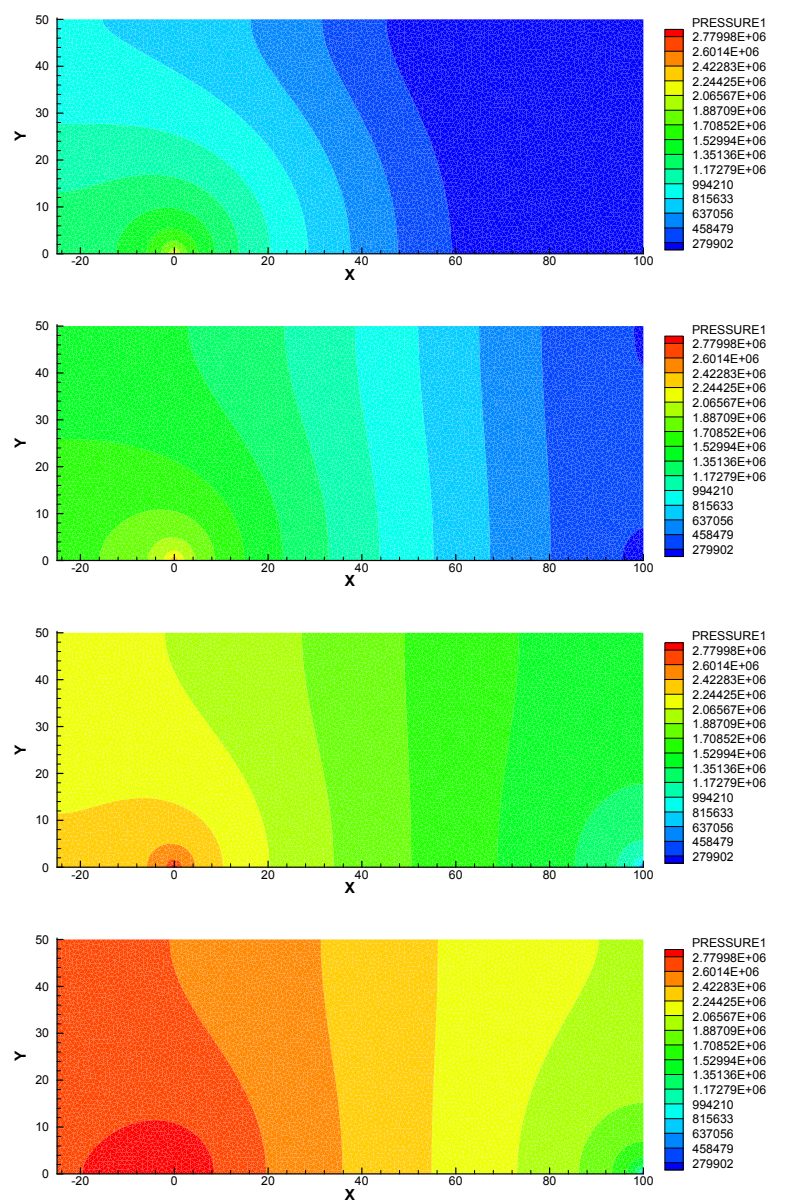

Fig.11 Evolution of air pressure distribution at $\mathrm{t}=5 \times 10^{6}, 10^{7}$, $2 \times 10^{7}$, and $3 \times 10^{7}$ seconds

\section{Table 2}

Fig. 8 depicts the temporal evolution of gas pressure at the observation point at the outlet. The numerical results of all implemented element types compare very well. Small deviation occur from different numbers of Gauss integration points.

\section{MODEL APPLICATION}

Heat transport in a soil layer is considered. The corresponding material properties are given in Table 1 and Table 2 except of saturated permeability,

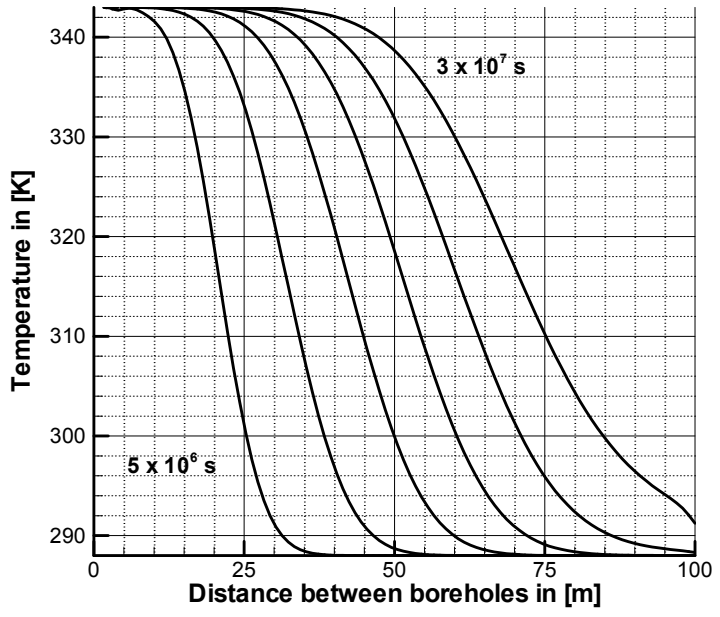

Fig.10 Temperature profiles between injection and extraction boreholes
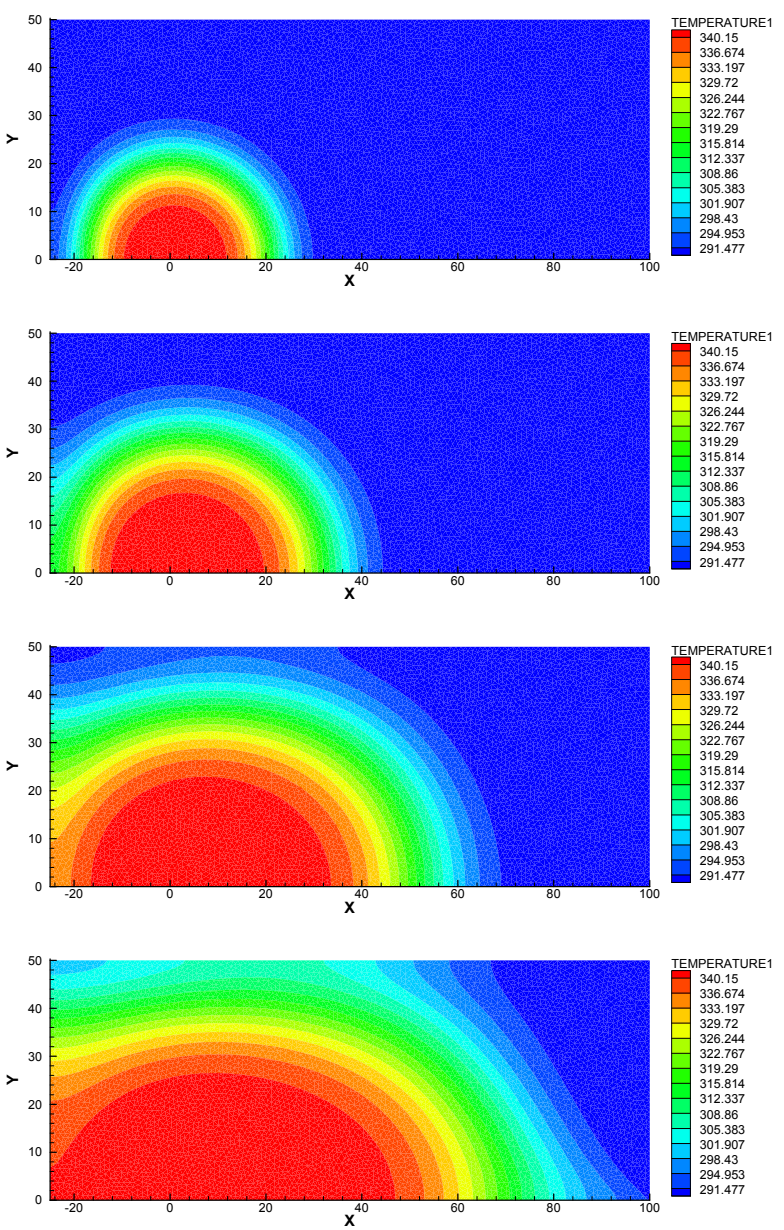

Fig.12 Evolution of temperature distribution at $\mathrm{t}=5 \times 10^{6}, 10^{7}$, $2 \times 10^{7}$, and $3 \times 10^{7}$ seconds

which is $k=2.7 \times 10^{-15} \mathrm{~m}^{2}$ typical for soils. We assume a constant layer thickness and that gravity effects can be neglected. Therefore, a 2-D horizontal model can be applied to this study. The distance between injection and extraction boreholes is $100 \mathrm{~m}$ (Fig. 9). A finite element triangulation is used (14562 
triangles). Fig. 9 illustrates the boundary conditions which are applied. We use no flow boundary conditions for flow and heat transport which means that the reservoir is hydraulically and thermally closed. A symmetry condition is applied along the borehole connection line.

Heated air $\left(70^{\circ} \mathrm{C}=343 \mathrm{~K}\right)$ is injected at 5 bar pressure into the soil layer. The initial temperature in the soil layer is assumed to be $15^{\circ} \mathrm{C}(=288 \mathrm{~K})$. The following sequence of Fig. 11 shows the evolution of air pressure distributions in the soil layer at $\mathrm{t}=58$, 116,232 , and 348 days, respectively. As air is a compressible fluid, transient flow is developing. As an example of pressure front propagation, the $1.5 \mathrm{bar}$ pressure isoline is reaching about $40 \mathrm{~m}$ after 58 days, $80 \mathrm{~m}$ after 116 days, and is arriving at the extraction borehole after 232 days (Fig. 11). At the end of the circulation time (348 days) a pressure level of about 3 bar is adjusted at the extraction well.

Fig. 10 depicts the temperature profiles between injection and extraction boreholes resulting from the transient flow of the compressible fluid (Fig. 11). It can be seen that the heated air is not arriving at the extraction borehole within 250 days.

Fig. 12 shows the evolution of the horizontal temperature field within the soil.

\section{CONCLUSIONS AND FUTURE WORK}

In this paper a numerical model is presented for non-isothermal flow of compressible fluids and heat transport in porous media. The non-isothermal flow and transport model accounts for temperature dependent material properties of fluids and the porous medium. The numerical model is verified against analytical solutions. Based on element object-orientation the numerical model is capable to treat every geometric finite elements which makes the code useful for a variety of practical applications 35). Element object-orientation means that the evaluation of element matrix calculation is generalized with respect to geometric dimension of element types (Fig. 7) and to matrix type, i.e. mass (fluid or heat), Laplace (conductivity and diffusion), advection and coupling types, see equations (20) and (25), respectively.

A simplified study for heat storage in a horizontal soil layer is presented in order to demonstrate the code features. The verified numerical model can be used now, e.g. for the assessment of the potential for heat storage in soils as well as the optimization of the operation of heat circulation systems in soils.

Despite the progress in the model and code development, the conceptual model has still some deficiencies such as the assumption of constant soil moisture and neglecting thermal phase change effects. This model improvements will be conducted in ongoing and future work.

ACKNOWLEDGMENT: This work was partly funded by the German Science Foundation (Deutsche Forschungsgemeinschaft, DFG) under grant (KO1591/2-3).

\section{REFERENCES}

1) T J Crowley. Causes of climate change over the past 1000 years. Science, 289(5477):270-277, 2000.

2) L Mastny. Vital Signs: The Trends That Are Shaping Our Future. Worldwatch Institute, Norton \& Company, 2005.

3) F Staiss, Ch Linkohr, and U Zimmer. Erneuerbare Energien in Zahlen -nationale und internationale Entwicklung. Technical report, BMU, Berlin, 2007. www.erneuerbare-energien.de.

4) JBW Day, JH Black, NA Chapman, and DH Hall. A physical model study of the storage of low-grade heat in unsaturated chalk. Journal of Hydrology, 56:61-83, 1982.

5) C Doughty, A Nir, and C F Tsang. Seasonal thermal energy storage in unsaturated soils: Model development and field validation. Technical Report LBL-29166, Lawrence Berkeley Laboratory, Berkeley, 1993. Report.

6) G S Bodvarsson. Mathematical modeling of the behaviour of geothermal systems under exploitation. Lawrence Berkeley Laboratory Report LBL13937., 27(10):417-421, 1982.

7) M Ettala, K Winqvist, and A Eerikinen. Principles and field experiments, in energy storage in unsaturated soil using pore air flow. Technical Report VTT Technical Research Notes 1389, Technical Research Center of Finland (VTT), Espoo, Finland, 1992. Report.

8) A Niemi, T Kling, M Kangas, and M Ettala. Heat transport in unsaturated zone thermal energy storage -analysis with two-phase and single-phase models. Transport in Porous Media, 51:67-88, 2003.

9) K Pruess. Tough-users guide. Technical Report LBL-20700-78, Lawrence Berkeley Laboratory, Berkeley, California, 1987. Report.

10) M Kangas. Modeling of Transport Processes in Porous Media for Energy Applications. PhD thesis, Helsinki University of Technology, Department of Technical Physics and Mathematics, Espoo Finland, 1996.

11) H D Voigt and M Lauterbach. Druckaufbaumessungen an Gas-Soden. Technical report, Zentrales Geologisches Institut, Berlin, 1985. Haefner et al. Geohydrodynamische Erkundung von Erdoel-, Ergas-und Grundwasserlagerstaetten.

12) M Muskat. The flow of homogenous fluids through porous media. J.W. Edwards Inc., Ann Arbor, Michigan, 1nd edition, 1937.

13) L S Leibenzon. Dvizhenie prorodnykh zhidkostei i gazov v poristoi srede (The flow of natural fluids in porous media). Gostekhizdat, 1947.

14) V I Aravin and S N Numerov. Theory of Fluid Flow In Undeformable Porous Media. Israel Program for ScientificTranslations, Jerusalem, 1965.

15) $\mathrm{P} C \mathrm{C}$ Johnson, $\mathrm{M}$ W Kemblowski, and $\mathrm{D} J$ Colthart. Quantitative analysis for the cleanup of hydrocarbon-contaminated soils by in-situ venting. Groundwater, 28(3):413-429, 1990.

16) D P McWorther. Unsteady radial flow of gas in the vadose 
zone. Journal of Contaminant Hydrology, 5:297-314, 1990.

17) A L Baehr and M F Hult. Evaluation of unsaturated zone air permeability through pneumatic tests. Water Resources Research, 27(10):2605-2617, 1991.

18) C Shan, R W Falta, and I Javandel. Analytical solutions for steady-state gas flow to a soil vapour extraction well. Water Resources Research, 28(4):1105-1120, 1992.

19) A L Baehr and C J Joss. An updated model of induced airflow in the unsaturated zone. Water Resources Research, 27(10):417-421, 1995.

20) C Shan. Analytical solutions for determining vertical air permeability in unsaturated soils. Water Resources Research, 31(9):2193-2200, 1995.

21) Y S Wu, K Pruess, and P Persoff. Gas flow in porous media with klinkenberg effects. Transport in Porous Media, 32:117-137, 1998.

22) E P Weeks. Field determination of vertical permeability to air in the unsaturated zone. US Geological Survey Professional Paper, (1051), 1987.

23) D E Wilson, R E Montgomery, and M R Sheller. A mathematical model for removing volatile subsurface hydrocarbons miscible displacement. Water Air Soil Pollution, 33(4):231-255, 1987.

24) A L Baehr, G E Hoag, and M C Marley. Removing volatile contaminants from the unsaturated zone by inducing advective air-phase transport. Journal of Contaminant Hydrology, 4:1-26, 1989.

25) C A Mendoza and E O Frind. Advective-dispersive transport of dense organic vapors in the unsaturated zone, 1. model development. Water Resources Research, 26(3):379-387, 1990.

26) K Pruess. Tough 2 -a general purpose numerical simulation for multiphase fluid and heat flow. Technical Report LBL-29400-78, Lawrence Berkeley Laboratory, Berkeley, California, 1991. Report.

27) R W Falta, K Pruess, I Javandel, and P A Witherspoon. Numerical modelling of steam injection for the removal of nonaqueous phase liquids from the subsurface, 1 . numerical simulation. Water Resources Research, 28(2):433-449,
1992.

28) P S Huyakorn, S Panday, and $\mathrm{Y} \mathrm{S} \mathrm{Wu}$. A three-dimensional flow model for assessing napl contamination in porous media and fractured media, i. formulation. Journal of Contaminant Hydrology, 16:109-130, 1994.

29) S Panday, P A Forsyth, R W Falta, Y S Wu, and P S Huyakorn. Considerations for robust compositional simulation of subsurface napl contamination and remediation. Water Resources Research, 31(5):1273-1289, 1995.

30) C I McDermott, A L Randriamanjatosoa, H Tenzer, and O Kolditz. Simulation of heat extraction from crystalline rocks: The influence of coupled processes on differential reservoir cooling. Geothermics, 35(3):321-344, 2006.

31) R C Reid, J M Prausnitz, and B E Poling. The properties of liquids and gases. McGraw-Hill, 1988.

32) A I Zografos, W A Martin, and J E Sunderland. Equations of properties as a function of temperature for seven fluids. Computer Methods in Applied Mechanics and Engineering, 61:177-187, 1987.

33) N B Vargaftik, J K Vinogradov, and V S Jar-gin. Handbook of physical properties of liquids and gases: Pures substances and mixtures. Begell House, Redding, 1996.

34) O Kolditz. Non-linear flow in fractured rock. Int $J$. Numerical Methods in Fluid and Heat Transport, 11(6):547-575, 2001

35) W Wang and O Kolditz. Object-oriented finite element analysis of thermo-hydro-mechanical (thm) problems in porous media. Int. J. Numerical Methods in Engineering, 69(1):162-201, 2007.

36) $\mathrm{R}$ Worsch. Numerical simulation of heat storage in the unsaturated zone. Diploma thesis, Center for Applied Geosciences, University of Tübingen.

37) P Grathwohl. Heat Diffusion. Lecture Scriptum, Center for Applied Geosciences, University of Tübingen.

(Received March 12, 2008) 